\title{
Childhood T Lymphoblastic Lymphoma
}

National Cancer Institute

\section{Source}

National Cancer Institute. Childhood T Lymphoblastic Lymphoma. NCI Thesaurus. Code C7210.

A T lymphoblastic lymphoma that occurs during childhood. 